\title{
Analysis of the Developmental Trend of Data Mining and the Applications on Precision Marketing
}

\author{
Yuanning Shi \\ Qinghai University of Finance and Economics College, QingHai, XiNing, 810016, China.
}

\begin{abstract}
Keywords: Data Mining, Precision Marketing, Applications, Developmental Trend, Feature.
\end{abstract}
\begin{abstract}
In this paper, we propose our research on the developmental trend of data mining and the applications on precision marketing. Internet community's greatest value lies in its unique interactive, also in the online community, share experience, Internet users to communicate with each other here, between business owners and consumers and potential consumers can realize the equal dialogue and communication, and realize the effective communication of information. Enterprises in implementing the precision on the basis of "discovery", take effective measures to strengthen the communication with consumers, its marketing effect is obvious and it is very effective. Our method integrate the core techniques of data analysis to propose the corresponding methodologies that is meaningful.
\end{abstract}

\section{Introduction}

Marketing theory and practice to some extent affected and restricted by the nature of marketing. To explore the essence of modern marketing is not only study the core of modern marketing theory, and is the guide of the enterprise market marketing activities. Marketing concept after one hundred years of development as has experienced several major changes, but whether it's idea about distribution, in the early or middle of exchange of views, or in the near future on the concept of value delivery, marketing is inseparable from the three basic elements [1-2].

To explore the essence of marketing, have to talk about the connotation of market marketing idea and marketing and human understanding of the marketing is growing, as the market marketing idea evolves, about the connotation and essence of marketing is increasingly clear. Enterprises as the main body of the marketing activities, marketing ethics is directly related to enterprise staff quality and the long-term development of the enterprise, to society, and also can produce certain effect to the country and if enterprise without good marketing ethics can trust of consumers will eventually be eliminated by the society. Under this requirement, we propose the principles of marketing as follows.

- Characteristics different from the general product marketing service marketing, and gradually became an independent field of marketing, and obtained the rapid development, it shows that the characteristics of the marketing method according to its marketing object difference that is gradually decomposition and deepen. Generally speaking, compared with tangible products, service has become, diversity, inseparability, cannot be stored and not transfer ownership etc.

- The starting point of green marketing and social marketing concept is also a certain degree of difference, the latter emphasizes corporate interests with long-term interest consumers and the society, but it does not pay attention to the social sustainable development.

- Relationship marketing, to the idea of system to analyze the enterprise's marketing activities, enterprise marketing activities as companies and general consumers, competitors, suppliers, distributors, government agencies and social organizations interaction process, the core of the marketing is correctly handle the relationship between enterprise and the individuals.

Enterprise network marketing to carry out the need to accurate analysis of customer group, through further subdivision of the target group, to identify the customer orientation, then the target group for the design of the specific marketing content, shape a more distinctive brand image, thus "accurate" to attract the attention of the target customer groups, rather than blindly for marketing. So that we can effectively improve the network marketing targeted, general enterprise limited marketing resources concentrated in the target customer group that changes in maximum marketing efficiency [3]. 
Based on the data to predict on the basis of price, will no doubt improve competitiveness and price strategy is one of the most flexible marketing mix strategy has many characteristics, such as visibility, can be quantified and dynamic, and at the same time is very important and can affect the enterprise's profit level and the product successfully to the market. Pricing strategy requires companies to develop competitive price, on fight for market share, to obtain position. Therefore, small and medium-sized enterprise strategic pricing plan should be: to determine the price of pricing objectives, price strategy, structure, price level, price and general promotion and so on entrepreneurial pricing factors.

\section{Details of Our Methodology}

Data Analysis and Marketing. The so-called digital marketing that is refers to using the Internet, computer communication technology and digital interactive media to achieve marketing goals of the marketing mode. This way of marketing give full play to the role of general modern communication technology, the whole process of marketing are under control of modern communication technology and computer technology that make the nervous around every corner of the product marketing of the enterprise to make enterprise marketing each terminal is full of the product marketing sensors, so as to change the information asymmetry between enterprises and marketing status to realize every product sales statistics with the predictable market changes.

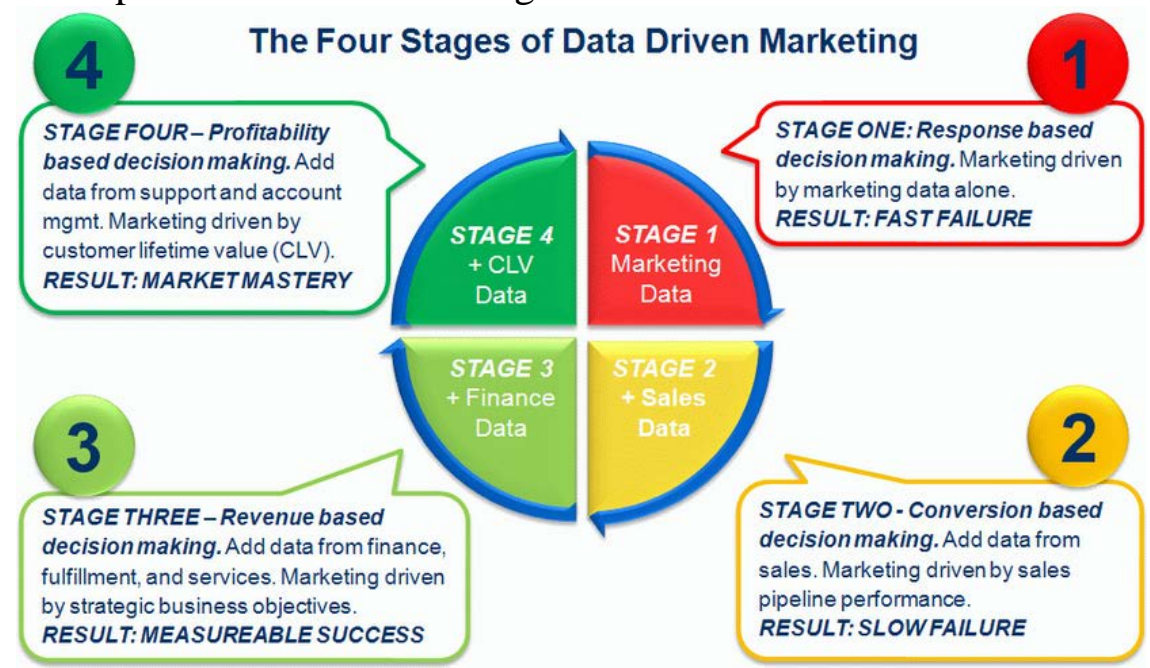

Fig. 1 The General Procedures of Data Analysis for Marketing

Big data in the data structure not only contains the transaction as representative of the traditional structured data that also contains a semi-structured data based on web information, and represented by multimedia information of unstructured data. From the perspective of the cross-selling with products and valuable customers that can start the marketing process but consider from the perspective of data mining, also need the product sales data and inventory data together, through the data analysis, to decide to increase or decrease of the goods all kinds of goods, to ensure that the correct inventory. Data warehouse system can also be inventory information and the general merchandise sales forecast information, via basic EDI system directly sent to the supplier, the supplier shall be responsible for the regular inventory, reduce the burden of the retailers [4].

The characteristics of big data marketing mainly manifested in the following respects. (1) Data driven marketing. With the continuous accumulation of raw data, big data platform has emerged, for marketers to better understand the customer provides a large amount of the information. (2) Forecast analysis ability. The accumulation of large data that marketers can through external and internal two system data for the analysis of current and future customer purchasing behavior. (3) Virtual activity ability. Through large data simulation can be some innovative people to test that the virtual market marketing idea, and in this virtual testing in the real market, the risk of cost saving. At the same time, because the data is used from the real world, therefore the virtual test conclusion has strong reliability. (4) Improve the personalization that mainly through the analysis of large data to more targeted to understand the needs of the customers, to provide customers with more personalized service. 
The Principles of the Marketing. Marketing concepts, the philosophy of marketing is a leap and revolution of germplasm, it not only changed the traditional way of old ideas of logical thinking, and it is a great breakthrough on the business strategy and method. It requires that the enterprise marketing management implementation "the customer is supreme" the principle of general management focus to discover and understand the needs of target customers, and one thousand ways to meet it, to achieve business goals. Therefore, the enterprise when deciding its production and operation must carry out market research, according to the market demand and choose target market condition of the enterprise itself, organization of general production operation, maximize customer satisfaction. The contents of marketing innovation in the new economic times can be organized as follows.

- The innovation of the marketing concept. The innovation of the marketing concept is the prerequisite and guarantee for the innovative activities of all other marketing. It throughout the course of enterprise marketing activities, and plays a leading role, directly affect enterprise marketing strategy formulation and implementation.

- Innovation of marketing method. Modern marketing to consumers as the center, to meet the needs of consumers, and consumers of the structure, preference, culture and so on the many factors that affect consumer behavior in the basic gradual change, the corresponding need to innovation. Marketing activities should balance the interests of the whole society.

- Marketing innovation. Market is one of the meanings of refers to the service object of the enterprise, as namely the target customers, it is characterized by a certain range of that some categories of reality and potential customers or collection of users, market innovation there are two basic ideas, one is to choose the sales area, the second is to choose to buy.

Reveal the connotation and essence of modern marketing as is a rational explanation, and exhibits the modern marketing nature humanization characteristics. To reveal the nature of modern marketing aim is not to replace individual goal, social goal but the universal under the guidance of the diversity; Rather than simply meet the demand of real customers, on behalf of the long-term interests.

Data Mining Steps. Specifically the so-called data mining in the database, data processing, from large, incomplete, noisy, fuzzy and the random data to extract basic implicit, previously unknown, but potentially useful information and knowledge of the process. Its object is a lot of day-to-day business data processing that can be reflected from the following figure two [5].

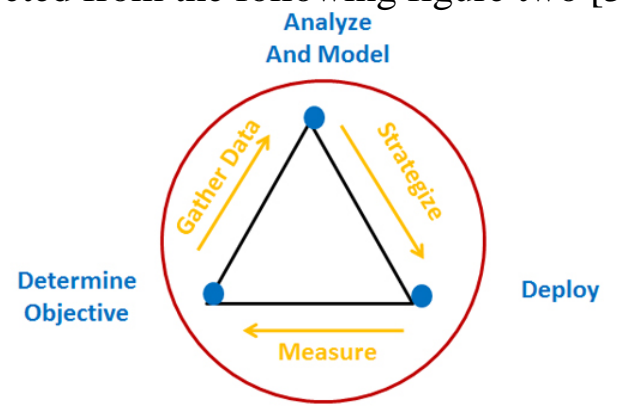

Fig. 2 The Systematic Architecture of the Data Mining Procedures

From the perspective of data analysis, data mining can be divided into two categories: describe the type of data mining and predictive data mining. The first with concise way to describe the data, and provides the general nature of interesting data. The latter analysis data, the establishment of one or a set of models, and try to predict the behavior of the new data set. Visual data mining technology is built on the basis of visualization and analysis process, it is to depict the functionality of the structure and display the data, and the tendency to the human perception mode, exceptions, and the ability to relationship as the foundation, to strengthen with visual data mining processing.

These huge amounts of data are often implies a variety of basic useful information, just rely on the database query retrieval mechanism and statistical methods are difficult to obtain this information, as urgently need to be able to automatically and intelligently convert data to be processed into valuable information, so as to achieve the aim of service for decision making. Accordingly, the procedures can be summarized as follows. (1) Data reduction techniques can be used to get data set reduction, said it was close to maintain the integrity of the original data, the data quantity is much smaller than the 
original data. Compared with the data reduction, on the reduction of the data mining, the required time and basic less memory resources, mining would be more efficient. (2) Need for data integration, data mining, that is, to combine data from multiple data sources in a same data storage, data source can be multiple databases, data cube or general data file. (3) By far the most commonly used method is to use the most likely value fill vacancies, such as tool can use regression, Bayesian formalism method and decision tree induction value to determine vacancy. These methods rely on existing vacancies by the data information of value, the value have much more chance to keep in touch with other attributes.

The Precision Marketing. The realization of the precision marketing, it is a system engineering. It includes detailed consumer demand analysis accurate market segmentation and positioning, precision marketing mix and precise marketing control and examination. Each other is independent each other, and depend on each other that form an inseparable whole. Network community has become after the portal and search another hot spot for the future development of China Internet network, based on its unique users and viscosity of the services, online community is increasingly strong marketing value are mining. The Internet is a technology-driven industries, the rapid development of science and the technology, network information changing, makes the network information is extremely complex and difficult to control. Enterprise does not need to also could not provide all the products in the market, meet the needs of all consumers, as long as you select one or several products direction and meet the needs of specific target consumer groups that can achieve the profit target of the enterprise, this is the meaning of market segmentation. If by reasonable positioning, can make the enterprise in the target market and shows a certain competitive advantage, and accepted and approved for the consumer, the enterprise to win in the starting line and the initial point.

\section{Summary and Conclusion}

In this paper, we propose our research on the developmental trend of data mining and the applications on precision marketing. Individuation tendency of consumer demand, poses challenges to enterprise the traditional marketing and the traditional marketing mode of "high investment, low returns" more disadvantages. Precision marketing, emphasizing the starting from consumers personalized needs, to take a targeted marketing strategy and proper combination, efficient marketing of target markets and customers, enhance customer satisfaction to reduce the marketing costs, achieve a win-win situation. We integrate data analysis techniques to enhance the traditional marketing that will be meaningful. In the future research, we will enhance the methodologies in advance.

\section{References}

[1] Stone, Merlin David, and Neil David Woodcock. "Interactive, direct and digital marketing: A future that depends on better use of business intelligence." Journal of Research in Interactive Marketing 8.1 (2014): 4-17.

[2] Li, Kai, and Timon C. Du. "Building a targeted mobile advertising system for location-based services." Decision Support Systems 54.1 (2012): 1-8.

[3] Huang, Jidong, et al. "A cross-sectional examination of marketing of electronic cigarettes on Twitter." Tobacco control 23.suppl 3 (2014): iii26-iii30.

[4] Lu, Wei, et al. "The bang for the buck: fair competitive viral marketing from the host perspective." Proceedings of the 19th ACM SIGKDD international conference on Knowledge discovery and data mining. ACM, 2013.

[5] Chaffey, Dave, and Mark Patron. "From web analytics to digital marketing optimization: Increasing the commercial value of digital analytics." Journal of Direct, Data and Digital Marketing Practice 14.1 (2012): 30-45. 